\title{
Familial cancers
}

NATHAN FREED, DO, FACOI

Stratford, New Jersey

Approximately $5 \%$ of all cancers have been recognized to have a pronounced genetic component. The most common examples of these include a nonpolyposis colon cancer family syndrome and certain familial breast cancers. The cancer family syndrome (CFS) includes the Lynch Syndrome Type I manifesting hereditary site-specific colon cancers (HSSCC) without extracolonic adenocarcinomas and the Lynch Syndrome Type II with extracolonic second primaries including endometrium and ovary. To meet the definition of CFS, they require early age of onset (between 40 and 50 ) and right-sided predominance. Analogously, it appears that breast cancer is hereditary in certain families, while by chance or for environmental reasons in others. Psychosocial issues stemming from familial cancers and the American Cancer Society Guidelines for screening in these circumstances will be discussed.

Some 50 hereditary cancers have been identified, ${ }^{1}$ and the number of recognized forms is growing steadily. Taken to completion, the final number may be between 100 and 1,000 of the 50,000 human genes thus far recognized. ${ }^{2}$ A number of large statistical surveys, in analyzing cancer incidence among relatives of cancer patients over the past five years, have revealed no more than a weak hereditary influence in most common adult malignancies. ${ }^{3}$ A small proportion of cancer patients (estimated at about $5 \%$ ) have been recognized to have a pronounced genetic component. ${ }^{4}$

Clinical relevance dictates that any brief overview such as this directs its attention to comparatively more frequent varieties. I will, therefore, focus my discussion on the heritable nonpolyposis syndromes that lead to colorectal cancer and additionally will discuss the familial breast cancers.

\section{Nonpolyposis colorectal cancer}

Familial colon cancers present two hereditary forms: (1) the mutation of one gene produces polyposis coli and a high risk of carcinomas, particularly in the descending sigmoid colon and rectum; and (2) a mutation of another gene accounts for the cancer family syndrome (CFS). As a group, the polyposis syndromes account for $1 \%$ to $5 \%$ of all colon cancers. ${ }^{5}$ Inherited nonpolyposis colon cancers occur with greater frequency and comprise up to a quarter of the colon cancers. ${ }^{5}$

CFS includes multiple anatomic sites, multiple primary malignant neoplasms, early age of onset, and autosomal dominant mode of inheritance. One of these is the Lynch syndrome II ${ }^{6}$ with its early onset of proximally located colonic cancer and extracolonic adenocarcinoma-particularly endometrial and occasionally ovarian cancer. Lynch syndrome $I,{ }^{6}$ or hereditary site specific colon cancer (HSSCC), has all of the same characteristics except extracolonic carcinoma. Families in the hereditary nonpolyposis colorectal cancer category (HNPCC $)^{5}$ exhibit the following characteristics: early age at onset of colorectal cancer; a greater incidence of proximal colonic cancer and increased frequency of multiple primary cancers, including carcinoma of the endometrium; and improve fiveyear survival of patients with colonic cancer who are compared by appropriate staging with the American College of Surgeons' long-term audit series.

Lynch and associates ${ }^{6}$ reported over 40 families (composed of some 3,000 members) with either a Lynch syndrome I or II profile. In a 1986 Finnish study, ${ }^{7,22}$ kindreds of CFS totaling 196 members were identified. Colorectal cancer was prevalent $(61 \%)$ in this study.

Patients who lack premonitory clinical signs or 
laboratory markers of hereditary colonic cancer are seen more frequently than their polyposis counterparts. Because only phenotypic expression alerts the physician to the genotype, it is very important to recognize high-risk patients early, while they are still potentially curable. The most vulnerable ages for expression of hereditary forms of colon and endometrial cancer are between 40 and 50 years. Early age of onset and right-sided predominant colon cancer among family members mandate the need for initiating colonoscopy at a young age in other family members. When clinical stigmata are lacking, one must rely heavily upon the pedigree for assessment of cancer risk status.

Correct clinical care of these cancer-prone families assumes that a thorough family history of cancer has been elicited. Questioning patients about first-line family members (parents, siblings, children) is key to finding syndromes restricted to direct-line relatives, as opposed to nonbloodline relatives. This argues for genetic inheritance and against environmental factors responsible for cancers observed. Second-degree relatives (grandparents, aunts, uncles, and grandchildren) should be included in the history taking. If it appears that several relatives have had tumors, it would be ideal to document their cases with medical records. The family might be referred to a medical geneticist, who could analyze mendelian, cytogenetic, and environmental factors and counsel persons at high risk. Death from cancer may then be prevented by alerting family members to their risk and expediting surveillance.

\section{Familial breast carcinoma}

A parallel situation to familial colon cancer is seen with hereditary form of breast cancer. In certain families, breast cancer alone is found at high frequency, while in other families, breast cancer and endometrial cancers cluster together. In still other families, breast carcinomas occur along with a diversity of other cancers, including soft-tissue sarcomas (Li-Fraumeni cancer family syndrome ${ }^{8}$ ) and central nervous system tumors.

It is well established that the presence of breast carcinoma in a woman's mother or sister increases her risk of the disease by two-to three-fold. Sisters and daughters of breast cancer patients diagnosed before menopause with bialteral tumors are at substantially increased risk; as relatives of breast cancer patients with unilateral tumors diagnosed late in life, they are at little or no increased risk.

If two affected sisters have premenopausal onset and one or both have bilateral disease, the lifetime risk to any other sisters or to a daughter of these patients is about $50 \%$. If mother and daugh- ter are premenopausal and one or both have bilateral disease, the risk to the daughter's sister or to her own daughter is $50 \%$. If mother and daughter are postmenopausal and each has unilateral breast cancer, the risk to the daughter's sister of female child is $16 \%$. If two sisters are postmenopausal and have unilateral disease, the risk among other sisters or their daughters is $7 \%$, which is similar to the normal sporadic incidence. ${ }^{9}$

Data derived from epidemologic studies consistently show a clustering of breast cancer in some families. It appears that breast cancer is hereditary in some families and occurs by chance in other families, while it is environmental in still others. ${ }^{10}$ Although some retrospective analyses suggest that familial breast cancers have a better prognosis than sporadic forms, ${ }^{11}$ accurate assessment awaits larger series and standardization by stage at presentation, age, and histology. ${ }^{12}$

\section{Psychosocial issues}

Individuals who seek genetic counseling for cancer need information as well as emotional support. Certain barriers to transfer of genetic information, including low self esteem, denial, fear, anger, guilt, enbarrassment, and insecurity, have been noted. ${ }^{9}$ These are often generated by the presence of cancer in a family member. An experienced counselor should know how much information to give family members at risk and when to give it, as well as when to wait and listen for signs of these obstructions. The physician seeking out members at risk must also be aware of these barriers and should refer to persons experienced in handling these situations when appropriate.

\section{American Cancer Society guidelines}

The American Cancer Society ${ }^{13}$ makes recommendations for appropriate screening of patients who are at normal or high risk for colorectal and breast cancer. For early detection of colorectal cancer, these include a digital rectal examination every year after age 40 , a stool blood test every year after age 50, and a proctosigmoidoscopic examination every 3 to 5 years after age 50 (following two annual examinations with negative results). These guidelines apply only to persons with no previous colorectal cancer history. Patients at high risk, including those in high-risk families, should be screened from an earlier age. For polyposis cancer family syndrome, specifically familial polyposis coli, Gebert and coworkers ${ }^{14}$ recommend proctosigmoidoscopy examinations beginning from age 10 in high-risk individuals with lifelong surveillance. If polyps are not initially found, sigmoidoscopic examinations should follow everyone for 2 
years or more. As to nonpolyposis coli cancer family syndromes, screening should begin $10-15$ years earlier than the ages recommended routinely by the American Cancer Society for normal risk patients.

With respect to breast cancer screening, the American Cancer Society ${ }^{15}$ recommends a mammogram every year for asymptomatic women aged 50 years and older and a baseline mammogram for those aged 35 to 39 years. Asymptomatic women 40 to 49 years of age should have a mammogram every 1 to 2 years, depending on physical and mammographic findings as well as other risk factors. In addition, a professional breast examination is recommended every 3 years for women aged 20 to 40 years, and every year for those older than 40 .
Women from high-risk families should have more regular breast examinations and mammograms from the time that is is realized that they are at high risk. Nulliparous women and those having first pregnancies beyond age 30 should be screened in a similar fashion as those who had familial breast cancer.

\section{Conclusions}

Primary care physicians must accept the responsiblity of seeking out those at high risk for hereditary colon or breast cancer. They also must offer or make available the best in up-to-date counseling in order to achieve the ideal goal, that is, improving the cure rate through earlier detection.
1. Knudson AG Jr: Hereditary cancer, oncogenes, and antioncogenes. Cancer Res 1985;45;1437-1443.

2. Knudson AG Jr: Model hereditary cancers of man. Prog Nucleic Acid Res Mol Biol 1983;29:17-25

3. Clemmesen J: Statistical studies in the etiology of malignant neoplasms: Review and results. Acta Pathol Microbiol Scand 1965;174(suppl): $1-543$

4. Knudson AG Jr: Genetics and cancer, in Burchenal JA, Octtgen HF (eds): Cancer achievements, challenges and prospects for the 1980's. New York, Alan R. Liss, 1981, pp 381-396.

5. Anderson DE: Familial predisposition, in Schottenfeld D, Fraumeni JF Jr (eds): Cancer epidemiology and prevention. Philadelphia, WB Saunders Co, 1982.

6. Lynch HT, Kimberling W, Albano, WA, et al: Hereditary nonpolyposis colorectal cancer (Lynch syndromes I and II): Clinical description of resource. Cancer 1985;56:934-938.

7. Mecklin JP, Jarvinen HJ, Peltokallio P: Cancer family syndromes: Genetic analysis of 22 Finnish kindreds. Gastroenterology 1986;90:328333.

8. Li FP, Fraumeni JF Jr: Prospective study of a family cancer syndrome. JAMA 1982;247:2692-2694.

9. Kelly PT: Genetic counseling with the cancer patient's family. Curr Probl Cancer 1983; 7(June):15-41.

10. King MA, Go Rc, Lynch HT, et al: Genetic epidemiology of breast cancer and associated cancers in high-risk families. JNCI 1983;71:463. 467.

11. Albano WA, Recabaren JA, Lynch HT, et al: Natural history of hereditary cancer of the breast and colon. Cancer 1982;50:360-363.

12. Birch JM, Hartley AC, Marsden HB, et al: Excess risk of breast cancer in the mothers of children with soft tissue sarcomas. $\mathrm{Br} J$ Cancer 1984;49:325-331.

13. Cancer-related checkups, publication no. 2070-LE. American Cancer Society, New York, 1984

14. Gebert, HF, Jagelman, DG, McGannon, E: Familial polyposis coli. Amer Fam Pract 1986;33:131-132.

15. Cancer facts for women, publication no. 2007-LA. American Cancer Society, New York, 1985

Dr. Freed is an associate professor of medicine and chief, Division of Hematology/Medical Oncology, University of Medicine and Dentistry of New Jersey - School of Osteopathic Medicine, Cherry Hill, New Jersey.

Dr. Freed, Ambulatory Health Care Center, 301 South Central Plaza, Suite 3100 , Stratford, New Jersey. 